Case Report

\title{
A Case of Coincidental Association of Amyotrophic Lateral Sclerosis in a Patient with Celiac Disease Consuming a Gluten-Free Diet
}

Edda Cava * , Alessandro Collo, Marcella Serioli, Sergio Riso

Unit of Dietetics and Clinical Nutrition, University Hospital "Maggiore della Carità", Novara, Italy; EMails: Edda.cava@maggioreosp.novara.it; Alessandro.collo@maggioreosp.novara.it; Marcella.serioli@gmail.com; Sergio.riso@maggioreosp.novara.it

* Correspondence: Edda Cava; E-Mail: edda.cava@maggioreosp.novara.it

Academic Editor: Luis Rodrigo

Special Issue: Gluten-Related Neurological Disorders

OBM Neurobiology

2020, volume 4, issue 4

doi:10.21926/obm.neurobiol.2004073
Received: June 30, 2020

Accepted: October 04, 2020

Published: October 12, 2020

\begin{abstract}
We report, for the first time, the case of amyotrophic lateral sclerosis (ALS) onset in a 54-yearold woman not following a strict gluten-free diet (GFD) for six years after the diagnosis of celiac disease (CD). The patient did not display any sign of gluten toxicity, malabsorption, or CDrelated comorbidity. Previous cases reported in medical literature showed ALS-like forms secondary to gluten intolerance, with symptom remission after a strict GFD. Even after correcting dietary flaws, the clinical manifestation of ALS did not improve in our patient. The main neurological involvement was a muscular weakness of upper and lower limbs, and dysphagia causing a weight loss for reduced food intake without any signs of malabsorption. Only vitamin D and folate levels were observed below normal; therefore, nutritional management required an oral supplementation of nutrients and vitamins. Muscle wasting was confirmed by an electrophysiological study using electromyography (EMG); body composition
\end{abstract}

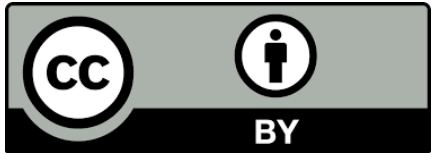

(C) 2020 by the author. This is an open access article distributed under the conditions of the Creative Commons by Attribution License, which permits unrestricted use, distribution, and reproduction in any medium or format, provided the original work is correctly cited. 
was assessed using bioimpedentiometry. Even after implementing a stricter GFD, clinical manifestations of the ALS did not improve. Therefore, the diagnosis of secondary neurological involvement in gluten ingestion was ruled out with ALS as an exclusionary diagnosis, at the end of the routine diagnostic pathway. The patient is currently under neurological and nutritional follow up for both ALS and CD as per standard of care to ensure her nutritional support and maintain the best possible quality of life during the progressive prognosis of the neurological diagnosis.

\section{Keywords}

Celiac disease; gluten-free diet; amyotrophic lateral sclerosis; gluten sensitivity; MarshOberhuber classification

\section{Case Report}

Amyotrophic lateral sclerosis (ALS) is a progressive motor neuron disease with degeneration of descending motor pathways. Its etiology and pathophysiologic mechanisms remain incompletely understood; therefore, ALS is often an exclusion diagnosis after ruling out different diseases, causing similar manifestations.

We report the case of a 54-year-old woman, not adhering to a gluten-free diet (GFD), showing ALS onset six years after being diagnosed with celiac disease (CD). She tested positive at serology (both IgA and IgG positive endomysium and anti-tissue transglutaminase, with normal anti-gliadin antibodies, and normal total IgA count), endoscopy, and histological tests (villous atrophy and crypt hyperplasia with lymphocytes infiltration of the mucosa at the duodenal biopsies that were staged $3 \mathrm{c}$ as per the Marsh-Oberhuber classification) for CD during her first pregnancy at 48 years of age, after three previous miscarriages. At that time, she had reported a $15 \%$ weight loss recorded in three months and a chronic iron deficiency; nevertheless, she, by following a strict GFD during the pregnancy, successfully delivered her baby. Thereafter, she was lost to follow-up and recently was referred to us. She started weekly exceptions to the GFD after delivery. She reported no other comorbidities, no family history of $C D$, and no other autoimmune or neurological diseases. Five years after the CD onset, she reported cervical rigidity, muscular weakness in upper and lower limbs. No fasciculation, cramp, or sensory loss was reported. She also displayed dysphagia for solid and liquid food, and pulmonary dysfunction with dyspnea on exertion, as measured by a spirometry test.

Routine examinations to confirm motor-neuron disease involved conducting laboratory tests, electrocardiogram, electromyography (EMG), abdominal ultrasound, magnetic resonance (MR) of the brain and spinal cord with fiber tracking diffusion tensor imaging (DTI).

Blood tests revealed an elevated creatine phosphokinase level, low level of Vitamin D, and folate without any other metabolically relevant abnormality (Table 1 ). The patient reported no other serological follow up during the preceding six years after implementing a GFD. 
Table 1 Laboratory results.

\begin{tabular}{|c|c|c|c|c|}
\hline & Reference values & $\begin{array}{l}\text { CD Diagnosis } \\
(2014)\end{array}$ & $\begin{array}{l}\text { CD Diagnosis } \\
(2019)\end{array}$ & $\begin{array}{l}\text { ALS diagnosis } \\
(2020)\end{array}$ \\
\hline RBC & $4-10 * 10^{3} / \mu \mathrm{L}$ & 4.46 & 4.81 & 4.61 \\
\hline $\mathrm{Hb}$ & $12-15.5 \mathrm{~g} / \mathrm{dL}$ & 11.7 & 13.7 & 12.9 \\
\hline $\mathrm{Ht}$ & $36-46 \%$ & 35.7 & 41.5 & 39.8 \\
\hline MCV & $82-98 \mathrm{fL}$ & 71 & 86.3 & 86.3 \\
\hline Iron & $50-170 \mu \mathrm{g} / \mathrm{dL}$ & 16 & 97 & 51 \\
\hline Transferrin & $250-380 \mathrm{mg} / \mathrm{dL}$ & 276 & 72 & 168 \\
\hline Ferritin & $10-291 \mathrm{ng} / \mathrm{mL}$ & 21 & 114 & 140 \\
\hline Proteins & $6.4-8.3 \mathrm{~g} / \mathrm{dL}$ & 6.7 & 6.1 & 6.7 \\
\hline Albumin & $3.4-4.8 \mathrm{~g} / \mathrm{dL}$ & 3.8 & 3.51 & 4.3 \\
\hline Glucose & $70-100 \mathrm{mg} / \mathrm{dL}$ & 68 & 91 & 84 \\
\hline Vitamin D & $30-100 \mathrm{ng} / \mathrm{mL}$ & 7 & 19.7 & 27.5 \\
\hline Vitamin B12 & $211-911 \mathrm{pg} / \mathrm{mL}$ & 213 & 340 & 375 \\
\hline Folate & $>3 \mathrm{ng} / \mathrm{dL}$ & $24^{*}$ & 3.4 & 15.73 \\
\hline Tot Cholesterol & $0-200 \mathrm{mg} / \mathrm{dL}$ & 162 & 177 & 172 \\
\hline Triglycerides & $45-170 \mathrm{mg} / \mathrm{dL}$ & 194 & 121 & 248 \\
\hline Total IgA & $70-400 \mathrm{mg} / \mathrm{dL}$ & 172 & & \\
\hline $\lg A$ tTg & $<20 \mathrm{CU}$ & 11 & 1.77 & 5.9 \\
\hline IgA AGA & $<20 \mathrm{CU}$ & 72 & 3.04 & \\
\hline IgG AGA & $<20 \mathrm{CU}$ & 5.4 & 3.87 & \\
\hline EMA & Positive/Negative & + & - & - \\
\hline $\begin{array}{l}\text { RBC Red Blood } \\
\text { Immunoglobulins } A\end{array}$ & ells; Hb Hemoglobi & . & $\begin{array}{l}\text { MCV Mean C } \\
\text { Endomysium A }\end{array}$ & $\begin{array}{l}\text { lular Volum } \\
\text { tibodies; BP }\end{array}$ \\
\hline
\end{tabular}

Other autoimmune neurological or rheumatological diseases, including heavy metal poisoning and endocrine disruption, were ruled out. No pathologic spinal cord disc conditions were found.

Both spinal and bulbar regions were involved at the EMG test with neuronal denervation, muscle weakness, and hyperreflexia. Lymph nodes were slightly swollen along the cervical spine without clinical relevance $(<2 \mathrm{~cm})$. A speech-language pathologist diagnosed dysphagia by a swallowing assessment, evaluating weakness of mouth, tongue, and facial muscle including pharyngeal phase impairment, voice impairment, drooling, and need for a modified consistency diet with a liquid thickening.

During the diagnosis of CD and the flawed GFD, the patient regained the lost weight at the CD onset and turned slightly overweight at ALS onset $\left(26.1 \mathrm{~kg} / \mathrm{m}^{2}\right)$. Unfortunately, her dysphagia was 
progressing rapidly, and during the next six months, the swallowing muscle weakness increased. She reported prolonged meal time and fatigue, restricted food intake recording a 10-12\% weight loss that required oral nutritional supplementation (ONS).

We also performed a bioimpedentiometric analysis showing marked sarcopenia for muscle wasting at the phase angle and body cellular mass (BCM), as shown in Figure 1 and Table 2.
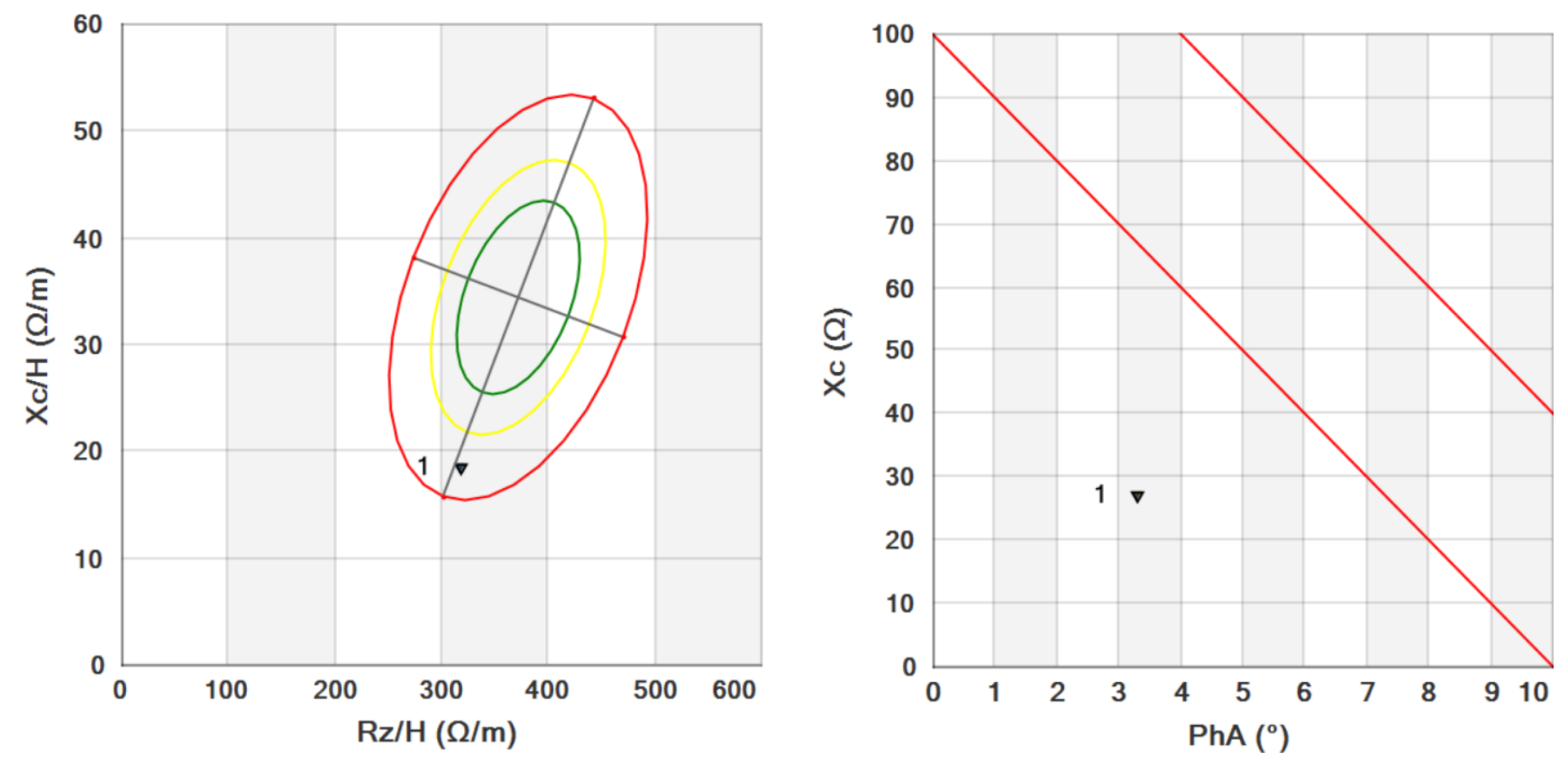

Figure 1 Bioimpedentiometry graph.

Rz Resistance; Xc Reactance.

Table 2 Body composition parameters.

\begin{tabular}{llll}
\hline & & Reference values & Units \\
\hline BMI & 26.2 & $18.0-25.0$ & $\mathrm{~kg} / \mathrm{m}^{2}$ \\
PhA & 3.3 & $5.6-7.4$ & $\circ$ \\
BCM & 9.5 & $>10.0$ & $\mathrm{~kg} / \mathrm{m}$ \\
FFM & 26.2 & $23.0-28.0$ & $\mathrm{~kg} / \mathrm{m}$ \\
FM & 12 & $7.0-14.0$ & $\mathrm{~kg} / \mathrm{m}$ \\
TBW & 27.1 & $15.0-22.0$ & $\mathrm{~L} / \mathrm{m}$ \\
\hline
\end{tabular}

BMI body mass index; PhA phase angle; BCM body cellular mass; FFM fat-free mass; FM fat mass;

TBW total body water. 


\section{Discussion}

Celiac Disease $(C D)$ is an intestinal enteropathy triggered by the ingestion of gluten in genetically predisposed individuals and leads to an autoimmune-mediated inflammatory response with duodenal villous atrophy and nutrients malabsorption. Typical presentation includes abdominal distension, diarrhea, steatorrhea, weight loss, iron deficiency anemia, and osteoporosis. Diagnosis relies on serological parameters (tissue transglutaminase and endomysial IgA) and duodenal biopsies (villous atrophy and increased intraepithelial lymphocytes) [1-2]. However, diagnosis is sometimes challenging due to atypical presentation, such as neurological symptoms (with or without gastrointestinal symptoms) including gluten ataxia, motor neuron disease-like neuropathy, small fiber type neuropathy, cognitive impairment, and Parkinsonism resulting from vitamin and mineral deficiencies.

A possible pathophysiologic role of anti-gliadin antibodies in a neurotoxic autoimmune process has been suggested in some previous studies [3-4]. Besides, other immunological disorders could overlap with $C D$, including Hashimoto thyroiditis, type 1 diabetes mellitus (T1DM), rheumatoid arthritis, and IgA deficiency [2].

An atypical presentation can even mimic signs and symptoms of ALS, such as severe, relentless, and progressive neurodegenerative motor neuron disease, resulting in increasing weakness of voluntary muscles until death from respiratory failure occurring within few years [5].

ALS is less frequent than CD (prevalence range in European population 1.1-8.2/100,000 vs. 1/100) with a later peak age at onset (58-63 years and 47-52 years for sporadic and familial forms, respectively, vs. a double peak in early childhood and the third-to-fourth decade, respectively). ALS etiology and pathogenesis remain undetermined, warranting further investigation of genetic causes and the contribution of environmental factors [6-7].

To our knowledge, the current case report is the first case of ALS reported in a patient with CD following a partially-compliant GFD. Previously reported cases showed an atypical presentation of CD, with neurological symptom onset classified as secondary ALS (ALS-like syndrome) that improved after a strict GFD implementation [2, 8-11].

Ham $\mathrm{H}$ et al. [9] reported an emblematic case of a sexagenarian man showing chronic progressive motor weakness within the last two years, highly suspected of having ALS. Since he reported loose stools and weight loss in the previous seven months, an esophagogastroduodenoscopy (EGD) was performed, and the histopathological examination revealed villous atrophy, crypt hyperplasia, and severe lymphocytic cells infiltration. According to higher serum endomysial antibody titer, the patient received a CD diagnosis, and after four months of GFD, the neurological manifestations showed an improvement.

Even Brown et al. [2] presented a case of a young man with progressive neurologic symptoms and brain MR imaging suggestive of ALS. They also diagnosed CD with neurological and imaging improvements through GFD. Other similar ALS-mimicking cases were reported [8-10].

ALS etiology is not completely known, and its relationship with CD remains still incompletely established. A higher prevalence of autoimmune associated diseases has been described [10, 12] in epidemiologic studies on ALS patients, but a causative relationship remains still to be established. 
Conversely, two earlier studies analyzing this relation showed no genetic correlation in a genomewide association study (GWAS) and established no serological evidence of gluten-related involvement in ALS etiology $[13,14]$.

Ludvigsson et al. [13] compared a large cohort of CD patients (diagnosed by small intestine biopsies) with the general population, finding no higher risk of ALS onset $(\mathrm{H} 1.0 ; 95 \% \mathrm{Cl}$ 0.5-1.8). Even CD-associated HLA allele frequencies were observed similar in ALS patients and controls, excluding any correlation between the two diseases [14].

Besides, a unique report [4] showed a higher frequency of seropositive ALS patients to the newly identified neuronal transglutaminase 6 (TG6) without manifesting any difference in the clinical neurological features of the disease. Since this report suggested an association of ALS with autoimmunity and gluten sensitivity, but data are still inconclusive, further investigations in this field are required.

Besides vitamin deficient status, an imbalance in redox species at a pathogenetic level was proposed as a possible link between autoimmune disorders and neurodegenerative diseases [15].

In our patient, the risk of developing an autoimmune disease might have been triggered by multiple exceptions to the GFD, but no evidence of a causal relation between CD and ALS has ever been described to support this hypothesis. On the other hand, an atypical neurological presentation of $C D$, defined as secondary ALS, could be ruled out as our patient re-started on stricter GFD after being diagnosed with ALS, and her symptoms did not improve accordingly. Indeed, laboratory blood tests at ALS onset showed complete remission of gluten-related impairments with negative serology for $C D$ and no vitamin deficiencies (Table 1). Therefore, the current report rules out the possibility of ALS getting triggered by sporadic gluten ingestion, and cannot elucidate the genetic correlation of ALS and $C D$.

While $C D$ has a benign prognosis due to the total remission of symptoms and intestinal lesions a few months after the beginning of a GFD, ALS is a rapidly progressive neurodegenerative disease associated with poor quality of life and fatal prognosis.

\section{Conclusion}

In summary, it is suggested to rule out the diagnosis of CD onset when presenting with atypical neurological symptoms. Patients require a thorough investigation, with CD-antibodies serological screen to assess CD or compliance with GFD and accurate analysis for malabsorption and nutritional deficiencies that might be related to the neurological manifestations.

No evidence of causal relation could be found on the basis of such a limited number of cases, but more research is suggested with a focus on individuals not strictly adhering to GFD to follow-up the risk of autoimmune disease, especially those not genetically related to CD-HLA, such as ALS.

\section{Acknowledgments}

We would like to thank the Neurology department for the collaboration with our Clinical Nutrition department in the multidisciplinary outpatient for amyotrophic lateral sclerosis. Patients are 
supported by multiple figures, among whom dieticians are very important to improve quality of life and sustain their nutritional requirements.

\section{Author Contributions}

EC and MS contributed to subject's clinical management and the idea to report the case; EC and AC contributed to write the manuscript and analyzing the data, EC AC MS and SR contributed to manuscript feedback, revision and final approval.

\section{Competing Interests}

Authors have no conflict of interest to disclose.

\section{References}

1. Gujral N, Freeman HJ, Thomson AB. Celiac disease: Prevalence, diagnosis, pathogenesis and treatment. World J Gastroenterol. 2012; 18: 6036-6059.

2. Brown KJ, Jewells V, Herfarth $\mathrm{H}$, Castillo $\mathrm{M}$. White matter lesions suggestive of amyotrophic lateral sclerosis attributed to celiac disease. Am J Neuroradiol. 2010; 31: 880-881.

3. Ihara M, Makino F, Sawada H, Mezaki T, Mizutani K, Nakase H, et al. Gluten sensitivity in Japanese patients with adult-onset cerebellar ataxia. Intern Med. 2006; 45: 135-140.

4. Gadoth A, Nefussy B, Bleiberg M, Klein T, Artman I, Drory VE. Transglutaminase 6 antibodies in the serum of patients with amyotrophic lateral sclerosis. JAMA Neurol. 2015; 72: 676-681.

5. Al-Chalabi A, Hardiman O. The epidemiology of ALS: A conspiracy of genes, environment and time. Nat Rev Neurol. 2013; 9: 617-628.

6. Couratier P, Corcia P, Lautrette G, Nicol M, Preux PM, Marin B. Epidemiology of amyotrophic lateral sclerosis: A review of literature. Rev Neurol (Paris). 2016; 172: 37-45.

7. Kiernan MC, Vucic S, Cheah BC, Turner MR, Eisen A, Hardiman O, et al. Amyotrophic lateral sclerosis. Lancet. 2011; 377: 942-955.

8. Bersano E, Stecco A, D'Alfonso S, Corrado L, Sarnelli MF, Solara V, et al. Coeliac disease mimicking amyotrophic lateral sclerosis. Amyotroph Lateral Scler Frontotemporal Degener. 2015; 16: 277279.

9. Ham H, Lee BI, Oh HJ, Park SH, Kim JS, Park JM, et al. A case of celiac disease with neurologic manifestations misdiagnosed as amyotrophic lateral sclerosis. Intest Res. 2017; 15: 540-542.

10. Turner MR, Chohan G, Quaghebeur G, Greenhall RC, Hadjivassiliou M, Talbot K. A case of celiac disease mimicking amyotrophic lateral sclerosis. Nat Clin Pract Neurol. 2007; 3: 581-584.

11. Wood H. Can gluten sensitivity mimic amyotrophic lateral sclerosis? Nat Rev Neurol. 2015; 11: 308.

12. Miller ZA, Rankin KP, Graff-Radford NR, Takada LT, Sturm VE, Cleveland CM, et al. TDP-43 frontotemporal lobar degeneration and autoimmune disease. J Neurol Neurosurg Psychiatry. 2013; 84: 956-962. 
13. Ludvigsson JF, Mariosa D, Lebwohl B, Fang F. No association between biopsy-verified celiac disease and subsequent amyotrophic lateral sclerosis--a population-based cohort study. Eur J Neurol. 2014; 21: 976-982.

14. Visser AE, Pazoki R, Pulit SL, Van Rheenen W, Raaphorst J, Van der kooi AJ, et al. No association between gluten sensitivity and amyotrophic lateral sclerosis. J Neurol. 2017; 264: 694-700.

15. Chiurchiù V, Maccarrone M. Chronic inflammatory disorders and their redox control: From molecular mechanisms to therapeutic opportunities. Antioxid Redox Signal. 2011; 15: 2605-2641.

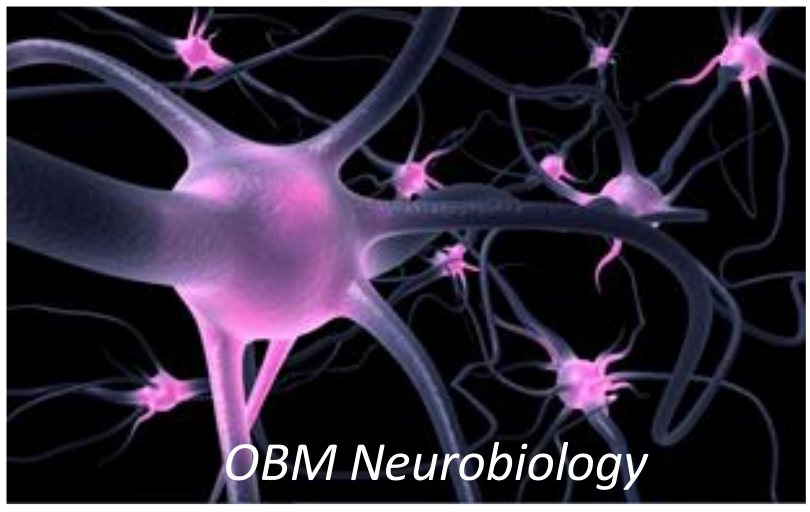

Enjoy OBM Neurobiology by:

1. Submitting a manuscript

2. Joining volunteer reviewer bank

3. Joining Editorial Board

4. Guest editing a special issue

For more details, please visit:

http://www.lidsen.com/journals/neurobiology 